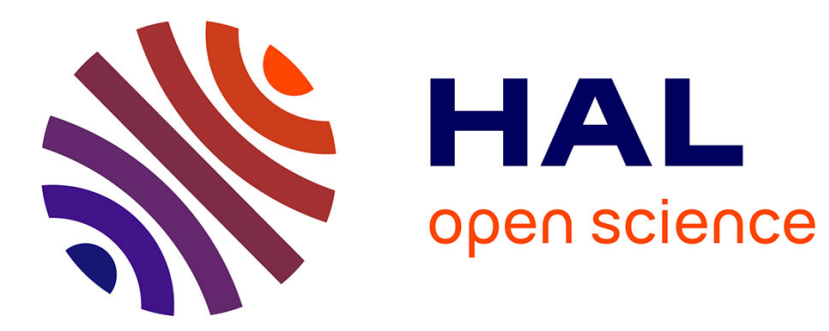

\title{
Global sensitivity analysis in welding simulations - what are the material data you really need?
}

Olivier Asserin, Alexandre Loredo, Matthieu Petelet, Bertrand Iooss

\section{To cite this version:}

Olivier Asserin, Alexandre Loredo, Matthieu Petelet, Bertrand Iooss. Global sensitivity analysis in welding simulations - what are the material data you really need?. Finite Elements in Analysis and Design, 2011, 47 (9), pp.1004-1016. 10.1016/j.finel.2011.03.016 . hal-00419162

\section{HAL Id: hal-00419162 \\ https://hal.science/hal-00419162}

Submitted on 25 Sep 2009

HAL is a multi-disciplinary open access archive for the deposit and dissemination of scientific research documents, whether they are published or not. The documents may come from teaching and research institutions in France or abroad, or from public or private research centers.
L'archive ouverte pluridisciplinaire HAL, est destinée au dépôt et à la diffusion de documents scientifiques de niveau recherche, publiés ou non, émanant des établissements d'enseignement et de recherche français ou étrangers, des laboratoires publics ou privés. 


\title{
Global sensitivity analysis in welding simulations - what are the material data you really need?
}

\author{
Olivier ASSERIN ${ }^{\mathrm{a}}$, Alexandre LOREDO ${ }^{\mathrm{b}, *}$, Matthieu PETELET ${ }^{\mathrm{a}, \mathrm{b}, 1}$, Bertrand IOOSS $^{\mathrm{c}}$ \\ ${ }^{a}$ CEA, DEN, DM2S, SEMT, LTA, F-91191, Gif-sur-Yvette, France \\ ${ }^{b}$ LRMA, EA 1859, Université de Bourgogne, France \\ ${ }^{c} C E A, D E N$, DER, SESI, LCFR, F-13108, Saint-Paul-lez-Durance, France
}

\begin{abstract}
In this paper, the Sensitivity Analysis methodology is applied to numerical welding simulation in order to rank the importance of input variables on the outputs of the code like distorsions or residual stresses. The numerical welding simulation uses the Finite Element Method, with a thermal computation followed by a mechanical one. Classically, a Local Sensitivity Analysis is performed, hence the validity of the results is limited to the neighborhood of a nominal point, and cross effects cannot be detected.

This study implements a Global Sensitivity Analysis which allows to screen the whole material space of the steel family mechanical properties. A set of inputs of the mechanical model - material properties that are temperature-dependent - is generated with the help of Latin Hypercube Sampling. The same welding simulation is performed with each sampling element as input data. Then, output statistical processing allows us to classify the relative input influences by means of different sensitivity indices estimates.

Two different welding configurations are studied. Considering their major differences, they give a different ranking of inputs, but both of them show that only a few parameters are responsible of the variability of the outputs. To prove it a posteriori for the first configuration, two series of computations are performed for a complete sample and for its reduced copy - where all the secondary parameters are set to mean values. They match perfectly, showing a substantial economy can be done by giving to the rest of the inputs mean values.

Sensitivity analysis has then provided answers to what we consider one of the probable frequently asked questions regarding welding simulation: for a given welding configuration, which properties must be measured with a good accuracy and which ones can be simply extrapolated or taken from a similar material? That leads us to propose a comprehensive methodology for welding simulations including four sequential steps: a problem characterization, a sensitivity analysis, an experimental campaign, simulations.
\end{abstract}

Key words: Sensitivity Analysis, material properties, random sampling, Finite Element, numerical experiments, welding simulation

\section{Introduction}

Control of mechanical effects of welding is a very difficult problem a lot of manufacturers have to solve, especially in the transport and the nuclear fields.

\footnotetext{
*Corresponding author

Email addresses: olivier.asserin@cea.fr (Olivier ASSERIN), alexandre.loredo@u-bourgogne.fr (Alexandre LOREDO), bertrand.iooss@cea.fr (Bertrand IOOSS)

${ }^{1}$ This work is part of a $\mathrm{PhD}$ thesis cofinanced by CEA (the French atomic energy agency) and the Burgundy French region

Preprint submitted to Elsevier
}

The Finite Element Method has been proved to be an effective tool for the welding simulation. The high increase in computer power allows nowadays simulating a complex welded assembly with a personal computer, in order to predict, from the conception stage, if mechanical behaviour is acceptable.

On the other hand, increasing processes complexity demands more and more accurate adjusting. Numerical simulation, provided that it can be sufficiently precise, is expected to become an important tool because it can considerably reduce the cost September 25, 2009 
of developments. For example, it allows optimizing parameters for special processes as for example in [1], more, it allows investigating new processes without any experimental device [2].

Hence, numerical simulation has to fulfil more and more requirements: control of mechanical welding effects like residual stresses and distortions, support to develop new processes, nuclear safety analysis reports, etc.

However, running a welding simulation requires inputs like mesh geometry, boundary and initial conditions, material properties and process parameters. The simulation generates several outputs, including spatial distributions of displacements and residual stresses in the seam. Among the aforementioned inputs, material properties are numerous.

This leads to one of the key problems of welding simulation. During a welding process, as temperature varies in a large amount, material properties vary strongly. Hence, they need to be inputted as arrays of 5 to 10 values for various temperatures instead of a single value. The number of input material parameters can rapidly reach high values, typically 50 to 100 .

It is quite difficult to use material data published in technical literature: firstly, it is sometimes difficult to find data for a given material apart from the classical ones; secondly, they are rarely characterized over a sufficiently wide temperature range. At high temperature, near the solidus temperature, most of these parameters are impossible to measure. More, some mechanical models involve parameters which are not present in classical data banks. On the other hand, for one given material, the full characterization is very expensive, often difficult or even sometimes impossible. For instance, it can take years to characterize a specific material without anyway knowing if all these data are significant.

To avoid this problem, numerical simulations are commonly done using available material data complemented with extrapolated values at high temperature and/or data given for a supposed similar material. This is not necessarily a bad method, because it will be shown in the present paper that, for a given problem family, some material properties are not very significant. The problem is to know what is permitted, in other words, the resulting inaccuracy induced by these practices.

Sensitivity analysis (SA) of these numerical models gives a rigorous answer to this question: it permits to notify what material properties need to be precisely determined and on the other hand, if it is possible to use a "mean value" or a "probable value" for some other properties.

Several studies regarding the influence of material properties in welding simulation are available [3, 4, 5, 6, 7, 8, 9, 10], but none of them can be considered as SA. Indeed, these parameters studies are only based on the comparison of results in terms of stresses and/or displacements of simulations considering different kinds of material properties evolutions with temperature. These authors have studied the influence of the thermo-mechanical material properties for various welding cases, with different material and models of material behaviour. Several papers seems to show that the distortions are mostly affected by thermal expansion and Young's modulus.

A study in which design variables are optimized to obtain minimum welding residual stresses can be found in [11]. Let us mention [12, 13] whose results tend to show that for the studied cases the distortions are mostly affected by thermal expansion and, to a lesser extent, by Young's modulus and yield strength. Schwenk [13] adds that Poisson's Ratio and the strain hardening have no noticeable influence on the calculated distortions and they can therefore be described by a master curve corresponding to the general alloy group. Moreover, this study shows that Young's modulus and the yield strength at high temperature don't have to be measured and can be taken from literature.

Sensitivity Analysis is also useful to solve inverse problem as in design optimisation. A very detailed review of Design Sensitivity Analysis methods can be found in [14] and a lot of later SA works covering various applications including welding can be found. Many of these approaches need to solve the adjoint problem, or involve partial derivatives which may be computed numerically by varying each input variable within a small interval around a nominal value and determining the corresponding effect on the output variable. However, few of these works deals with welding modelling and influence of material properties is not explicitly studied.

In welding simulations, the works cited above are based on local SA, which is of limited use for several reasons: (i) the validity of the results is limited to the neighbourhood of the studied material(s); (ii) in consequence the results of a local SA in welding simulation don't provide for an exploration of the rest of the material space of the input factors, i.e. it is improper, on the basis of a local SA, to 
make general recommendations on which material properties are necessary to know to perform an accurate simulation; (iii) local SA is justified to assess the relative importance of input factors only if the output behaviour is proved to be linear.

Global SA, on the opposite, avoids these problems. In the two last decades, the work of searchers like Morris [15], Helton [16], Saltelli [17], Kleijnen [18], Iooss [19] helped to promote the use of global SA. Practical aspects of global SA and its applications can be found in the following books 20 , 21, 22, 23]. This methodology takes into account the entire variation range of all inputs, and tries to apportion the output variation between them. This technique has been successfully used in a large variety of domains.

In this work, complete methodology of the global SA is successfully applied to welding simulation. The input space concerns only the mechanical properties and is extended to all steel materials. This leads to a quantitative classification of the most important parameters. To use this technique, it was necessary to develop specific sample techniques and choose a relatively light problem with the aim to perform a lot of simulations within a reasonable time.

The present paper is organized as follows. In Section 2, the mathematical modelling of mechanical effects of welding is discussed, firstly with the thermal model and secondly with the elasto-viscoplastic mechanical model. In Section 3. we describe the global sensitivity analysis by computations of correlation coefficients between input and output variables and we propose a technique for generating Latin Hypercubes samples with a monotonic dependence. Samplings and statistical analysis were realized with the SSURFER toolbox 24] developed within the $\mathrm{R}$ Software [25]. In the last part of this work, the global SA is performed on two different welding studies.

\section{Finite element model}

The welding thermo-mechanical problem is solved by the Finite Element Method. In welding problems, the thermal history has a notable influence on the mechanic behaviour because firstly, temperature is directly responsible to the thermal expansion which gives strains and then stresses, and secondly temperature affects mechanical properties like Young's modulus or the yield strength. On the other hand, the reverse effect is negligible: the heat produced by mechanical nonlinearities like plasticity is very low compared to the one delivered by the welding source. This kind of problem is said to be weakly coupled.

According to this fact, people generally simulate welding within two times: first the thermal field time evolution is computed solving the timedependent heat conduction equation; secondly, the mechanical equilibrium equations are solved for each time step, the thermal field, which had been previously computed for this time, token as a thermal load. For the mechanical problem, time is not explicitly present (there is no dynamical effects) but the elasto-visco-plastic behaviour creates an evolution which must be stored and updated during the nonlinear solving iterations. Outputs of the mechanical problem are classically the displacement, strain and stress field histories. For practical needs, displacements and stresses of the last time step, when the process is supposed to be finished, are of particular interest.

\subsection{Thermal computation}

As mentioned before, the thermal field history is computed solving the time-dependent heat conduction equation. Heat conduction is assumed to obey to Fourier's law, with a temperature-dependent heat conductivity.

The complex phenomena of heat deposit occuring during welding operation, including phase changes, magnetic effects and fluid dynamics are not modelled here. The thermal deposit is modelled by means of a parametric volumetric function. This approach is classically considered to be sufficient to deal with mechanical effects of welding. Once the function is chosen, values of its parameters can be calibrated for example by inverse methods based on experiments.

Heat losses are classically convection and radiation losses. They are generally relatively low compared to the heat provided by the source.

So, the problem to be solved is:

$$
\rho C_{p} \dot{T}-\nabla \cdot(\boldsymbol{\lambda} \nabla T)-Q(t)=0 \quad \text { in } \Omega
$$

where $\rho$ is the density, $C_{p}$ the specific heat capacity, $\boldsymbol{\lambda}$ the thermal conductivity tensor, $T$ the temperature, $Q$ the volumetric heat source and $t$ the time. The upper dot in $\dot{T}$ denotes the time derivative of $T$ and $\Omega$ is the space domain.

The domain boundary is classically split into two parts $\partial \Omega=\partial \Omega_{T} \cup \partial \Omega_{q}$ with $\partial \Omega_{T} \cap \partial \Omega_{q}=\emptyset$ to 
take into account prescribed temperature (Dirichlet) conditions and heat flux exchanges (Neumann) conditions:

$$
\begin{aligned}
& T=T_{p}(t) \quad \text { at } \partial \Omega_{T} \\
& \boldsymbol{\lambda} \nabla T \cdot \boldsymbol{n}=q(T, t) \quad \text { at } \partial \Omega_{q}
\end{aligned}
$$

where $\partial \Omega_{T}$ (respectively $\partial \Omega_{q}$ ) is the part of the boundary receiving prescribed temperatures $T_{p}(t)$ (respectively heat flux exchanges $q(T, t)$ ) and $\boldsymbol{n}$ is the outward unit normal to $\partial \Omega$.

For metal welding simulation, it is possible to take into account the heat flux provided by the welding source by means of a volumetric heat source $Q(t)$ in equation 1 or by means of a surface heat source $q(t)$ in equation 2. Exchanges at the boundaries include losses by convection and radiation according to the classical formulae:

$$
\begin{aligned}
& q_{c o n v}=h(T)\left(T-T_{0}\right) \\
& q_{\text {rad }}=\xi \sigma\left(T^{4}-T_{0}^{4}\right)
\end{aligned}
$$

where $T_{0}$ is the ambient temperature, $h$ is the convective heat transfer coefficient, $\sigma$ is the StefanBoltzmann constant $\sigma=5.67 .10^{-8} \mathrm{Wm}^{-2} \mathrm{~K}^{-4}$, and $\xi$ is the material surface emissivity. Boundary conditions are then defined by equations (2), where $q(T, t)$ includes the sum of $q_{c o n v}$ and $q_{\text {rad }}$ and if a surface type source is adopted - $q(t)$.

In order to take into account heat involved in phase changes in the vicinity of the welding source path (metal is melted, then solidified as the source goes forward) a standard method is used: an effective specific heat based on the local slope of the enthalpy is computed and introduced into the unsteady nonlinear solving algorithm.

This model does not take into account the precise mechanism of heat deposit which differs strongly according to the welding process type (Laser welding, Gaz Tungsten Arc Welding, Electron Beam Welding). It does not either take into account the heat convection caused by melted metal flow occurring in the welding pool. It is possible to augment the thermal conductivity $\lambda$ of the material for temperatures higher than the melting point to represent this phenomenon, but this is a poor way to do it: the welding pool shape might then be badly estimated, and it is precisely in the melted zone that mechanical stresses are set to zero before the cooling down and its thermal contraction. However, these two points are not of crucial importance for our concerns, because a source tuned by inverse method based on experiments [26] was considered.
Despite of the various phenomena involved, the nonlinear behaviour and the time-dependent nature, this problem is a quite classical one Finite Element codes are generally able to solve, provided a pertinent choice of algorithm and convergence criteria is done.

\subsection{Mechanical computation}

The mechanical equilibrium equation is:

$$
\sigma_{j i, i}+f_{j}=0
$$

where $\boldsymbol{\sigma}$ is the second order Cauchy stress tensor and $\boldsymbol{f}$ the body forces per unit volume.

The finite element program solves this equation by means of a weak form of the problem, which leads to a displacement formulation. In other words, the discretised structure behaviour is entirely described by its nodes displacements. The displacement field $\boldsymbol{u}$ is related to the strain tensor $\boldsymbol{\epsilon}$ by the classical small strains relation:

$$
\epsilon_{i j}=\frac{1}{2}\left(u_{i, j}+u_{j, i}\right)
$$

Stresses $\sigma_{i j}$ are related to strains, in a simple way only when linear elasticity is considered. Unfortunately, this is not the case here. The paragraph below is devoted to expose the particular mechanical behaviour considered in this study.

\subsubsection{Mechanical behaviour model}

The mechanical part of metal welding simulation is a difficult problem because several phenomena are to be considered. Of course, the thermal expansion, which is the load applied to the structure, is of primer importance. A large part of the structure goes under elastic deformation. Elastic behaviour depends generally on temperature, which is a first cause of non-linear behaviour.

In some areas, the yield strength can be reached and plastic strain occurs. This is helped by the classical lowering of the yield strength at high temperature. This plastic deformation can be modelled given a plasticity criterion, a flow rule, and a hardening rule - if any.

According to the material type, the temperature range and the speed of deformation, a viscous behaviour can be observed. Then the speed of deformation and the stresses are no longer independent. This phenomenon must be taken into account if necessary. 
Of course welding implies melting: in the melting zone, all the strains and stresses are set to zero. When the welding source goes away, the metal solidifies, and a thermal contraction begins, itself involving all phenomena described above.

In addition, some materials are subject to phase changes with temperature, causing local expansion or contraction, and then strains.

All these phenomena are taken into account assuming the classical strain decomposition:

$$
\epsilon_{i j}=\epsilon_{i j}^{e l}+\epsilon_{i j}^{t h}+\epsilon_{i j}^{p}+\epsilon_{i j}^{v p}+\epsilon_{i j}^{p t}
$$

with:

$\epsilon_{i j}^{e l}:$ elastic part of strain,

$\epsilon_{i j}^{t h}:$ thermal part of strain,

$\epsilon_{i j}^{p}:$ plastic part of strain,

$\epsilon_{i j}^{v p}$ : viscoplastic part of strain,

$\epsilon_{i j}^{p t}$ : phase transformation part of strain.

- $\boldsymbol{\epsilon}^{e l}$ : the elastic part of strain, is related to the difference between the actual stress $\boldsymbol{\sigma}$ and the initial stress tensors $\sigma^{I}$, by the compliance tensor $\mathbf{S}(T)$ which is the inverse of the fourth-rank stiffness tensor $\mathbf{C}(T)$ :

$$
\epsilon_{i j}^{e l}=S_{i j k l}(T)\left(\sigma_{k l}-\sigma_{k l}^{I}\right)
$$

For an isotropic material, the compliance tensor $\mathbf{S}(T)$ depends on only two parameters, it can be written for example in terms of Young's modulus $E(T)$ and Poisson's ratio $\nu(T)$.

- $\boldsymbol{\epsilon}^{\text {th }}$ : the thermal strain, which increments depends on the variation of the temperature $d T$ and on the thermal expansion tensor which is spherical in the isotropic case and then depends only on the scalar thermal expansion coefficient $\alpha(T)$ :

$$
d \epsilon_{i j}^{t h}=\alpha(T) \delta_{i j} d T
$$

- $\epsilon^{p}$ and $\epsilon^{v p}$ : the plastic and viscoplastic parts of strain depends on the chosen material behaviour. Elasto-plasticity models with kinematic hardening are able to represent behaviour of metals for temperatures lower than one quart of their melting temperature in Kelvin. For higher temperatures, and especially for temperatures near the melting point, the viscosity phenomenon appears and elasto-viscoplastic models are better. In this study, the first example uses a classical elastoplastic model with linear kinematic hardening which needs 5 material parameters; the second one uses Chaboche's elasto-viscoplastic model with nonlinear kinematic hardening 27], which needs 8 material parameters. This last model is detailed below. It is possible to envisage the use of both models in the same study (both models working on different zones) but this is not the case here.

- $\epsilon^{p t}$ : the phase transformation part of strain. When phase transformation - like the martensitic one- occurs, changes in density of a part of the material creates distortions which can be handled using this specific strain part. Its relative importance depends on the considered materials. In this study, only the melting phase transformation is considered, so this part of strain does not appear in this model.

Hence, for our concerns:

$$
\epsilon_{i j}=S_{i j k l}(T)\left(\sigma_{k l}-\sigma_{k l}^{I}\right)+\epsilon_{i j}^{t h}+\epsilon_{i j}^{(p / v p)}
$$

the $p$ or $v p$ exponent is selected according to the chosen model and to the remark formulated before.

\subsubsection{Elasto-viscoplastic model with non-linear kinematic hardening}

In this mathematical model, the stress tensor is allowed to take values in a finite domain of the sixth-dimensional stress space. This domain is bounded by a yield criterion. For metals, Von Mises's criterion is considered as a good model. It is written forcing Von Mises's stress $\sigma_{v}=J_{2}(\boldsymbol{\sigma})=$ $\sqrt{3 s_{I I}}$ to remain lower than the yield strength $\sigma_{y}$. $s_{I I}$ is the second invariant of the deviatoric stress tensor $s$, both defined as follows:

$$
s_{I I}=\frac{1}{2} s_{i j} s_{i j} \quad \text { with } \quad s_{i j}=\sigma_{i j}-\frac{\sigma_{k k}}{3} \delta_{i j}
$$

Here, a kinematic hardening model is used, which allows the viscoelastic domain to translate, hence to have a centre $\boldsymbol{\sigma}^{b} \neq \mathbf{0}$, usually called backstress tensor 2 Hence, the yield criterion becomes:

$$
f\left(\boldsymbol{\sigma}, \boldsymbol{\sigma}^{b}\right)=J_{2}\left(\boldsymbol{\sigma}-\boldsymbol{\sigma}^{b}\right)-\sigma_{y}
$$

\footnotetext{
${ }^{2}$ The backstress tensor is usually denoted $\mathbf{X}$, notation reserved in this study for the input data matrix.
} 
If the stress tensor remains in the domain, i.e. $f\left(\boldsymbol{\sigma}, \boldsymbol{\sigma}^{b}\right) \leq 0$, the behaviour is elastic. If it reaches the boundary of this domain, i.e. $f\left(\boldsymbol{\sigma}, \boldsymbol{\sigma}^{b}\right)=0$, plastic strain occurs. The plastic strain obeys to a flow rule: the plastic strain increment is assumed to be normal to the domain boundary at the stress location:

$$
\dot{\epsilon^{p}}=\dot{p} \frac{d f}{d \boldsymbol{\sigma}}
$$

and occurs with a velocity given by an expression corresponding to Norton's (or Odqvist's) law:

$$
\dot{p}=\left\langle\frac{f}{K}\right\rangle^{n}
$$

where $\langle$.$\rangle are the McCauley brackets which gives the$ value of its argument if this argument is positive, and zero otherwise. The viscoplastic exponent $n$ and the viscoplastic reference stress $K$ are material parameters, both depending on temperature.

The elastic domain center $\boldsymbol{\sigma}^{b}$ moves following the hardening rule:

$$
\dot{\boldsymbol{\sigma}}^{b}=\frac{2}{3} C A \dot{\boldsymbol{\epsilon}}^{p}-C \boldsymbol{\sigma}^{b} \dot{p}
$$

where $A$ and $C$ are material hardening parameters.

The elastic part of the strain tensor is linked to the stress tensor by Hooke's law:

$$
\sigma_{i j}=C_{i j k l}(T)\left(\epsilon_{k l}-\epsilon_{k l}^{t h}-\epsilon_{k l}^{(p / v p)}\right) ;
$$

Hence, this model needs the eight following material characteristics: Young's modulus E, Poisson's ratio $\nu$, the thermal expansion coefficient $\alpha$, the yield strength $\sigma_{y}$, hardening coefficients $A$ and $C$, the viscoplastic exponent $n$ also called rate sensitivity coefficient and the viscoplastic reference stress $K$ also called viscoplastic resistance.

\subsubsection{Material characteristics measurements}

Because of the complexity of this mechanical model, experimental measurements of these material characteristics is a difficult task, especially at high temperatures (for many it is almost impossible above $1200^{\circ} \mathrm{C}$ ). Complete set of data are only known for a little number of materials. However, the limits of the variations of theses parameters within a material family like the steel family for example can be fixed. This will lead to a domain, which will be the starting point of sensitivity analysis.

\subsubsection{Boundary conditions}

It is well known that boundary conditions can affect considerably the mechanical response of the structure. A quasi-free structure and, at the opposite, a strongly clamped one, lead to different displacements and stress fields. This is true for real experiments and obviously for numerical studies. In fact these boundary conditions can vary strongly with the nature of the problem, and there is no rule to apply. A careful examination of the real system and a solid experience of such problems are necessary to make a good choice...

\subsubsection{Cut-off temperature}

In [28] Lindgren made a classification of welding numerical simulations according to different criteria. One of these criteria is the use of a cutoff temperature $T_{c u t}$, above which the changes on the mechanical material properties are not taken into account (properties are considered constant from $\left.T_{\text {cut }}\right)$. The use of a cut-off temperature permits to avoid numerical problems encountered with very low properties values. For example, concerning Young's modulus, for temperatures higher than $T_{\text {cut }}$, the considered constant value must be low enough to ensure that the welding pool can not transmit significant stresses, but high enough to avoid the ill-conditioning of the stiffness matrix.

\subsubsection{Concluding remarks on the mechanical mod- elling section}

The mechanical model is more complex to handle than the thermal one. It is non-linear for several reasons. The material properties are non-linear, but this is relatively easy to consider. The main difficulty lies in the fact that the plasticity / viscoplasticity strains are cumulative and depends on stresses, temperature, speed. . The strains must be integrated to give the displacement field on which the Finite Element Method is based. All this computation needs time control, iterative schemes, convergence supervision, etc. which details go outside the purpose of this study.

Nevertheless, these procedures are classically managed by the actual softwares, and these complex simulation can be handled by a correctly trained user.

\subsection{Concluding remarks on the modelling section}

The computation is made in two stages: first the unsteady thermal computation is made, taking into 
account the required properties of the material and a heat source tuned by comparison with experiment. As the thermal part of the global computation was not scheduled to be considered in the sensitivity analysis, the thermal field history was stored and taken as the load for the second part of the computation, the mechanical one. The mechanical models used are the elasto-plastic with linear kinematic hardening (first study) and the Chaboche elasto-viscoplastic model with non-linear kinematic hardening (second study). The first model is governed by 5 material properties each of them with a temperature dependence which leads to a choice of a 7 points discretisation, while the second is governed by 8 material parameters, with a lighter choice of a 5 points discretisation. These $M=35$ (or 40) parameters are the input parameters of the sensitivity analysis.

\section{Sensitivity analysis}

Sensitivity analysis (SA) is the study of how the variation (uncertainty) in the output of a mathematical model can be apportioned, qualitatively or quantitatively, to different sources of variation (uncertainty) in the input of a model [29].

\subsection{The model, inputs and output(s)}

Here, the mathematical model is the welding numerical model, inputs have been introduced previously but the output(s) has to be specified.

Classically, the welding numerical simulations give as results a large number of values: time evolutions of the displacement, strain and stress fields over the entire structure. This can easily represent $10^{6}$ or $10^{7}$ real numbers. In practise, for a given study, few of them are used - except for maps and curves drawing. They are sometimes combined to give some macroscopic information. The variety of welding studies lead to focus on different kind of results: a single value, for example the displacement of a given node at the end of the experiment, or the maximum displacement of a given node during the experiment, or a maximum stress; a function of several values, for example the total elastic energy stored at the end of the experiment, etc. To process to a meaningful and effective sensitivity analysis, it is then necessary to choose a (a few) representative output(s) for the considered study. For simplicity, the following presentation is restricted to a single output.
Once the choice of inputs and output is done, mathematically, the numerical model can be regarded as a function $f$ with $M$ real arguments $x_{j}$ (or a $M$-dimensional vector $\mathbf{x}$ ) which gives a real value - the output- $y$ :

$$
\begin{aligned}
f: & \mathbb{R}^{M} \\
& \rightarrow \mathbb{R} \\
& \mapsto y=f(\mathbf{x}),
\end{aligned}
$$

The function $f$ represents the behaviour of the numerical model, which is of course not explicitly known.

To fix the ideas, the number $M$ of input parameters of studies presented below is 35 for the first one and 40 for the second, numbers which are relatively large for a sensitivity analysis. Moreover, the sensitivity analysis is done for 4 different outputs in the first study and for 3 similar outputs for the second one, see the section 4 for more details.

\subsection{Classification - Local Sensitivity Analysis versus Global Sensitivity Analysis}

We choose here to follow the classification proposed by Saltelli [20]: sensitivity analysis methods are classified into three groups, Screening methods, Local Sensitivity Analysis methods and Global Sensitivity Analysis methods.

The Screening methods are of practical interest because of their simplicity and their low cost but give only qualitative results (see the chapter 4 of the book [20]). The characteristics of the two other groups are briefly present below. The Local Sensitivity Analysis (LSA) studies how little variations of input parameters around a given value change the value of the chosen output. This involves partial derivatives, which may be computed numerically by varying each input variable - one at a time (OAT) — within a small interval around a nominal value and determining the corresponding effect on the output variable. The LSA, in its classical form, consists in computing the $M$ partial derivatives:

$$
S_{j}={\frac{\partial f}{\partial x_{j}}}_{\mid \mathbf{x}=\mathbf{x}^{0}}
$$

The local sensitivity is often normed by the local values $x_{j}^{0}$ and $y^{0}$ :

$$
S_{j}=\frac{x_{j}^{0}}{y^{0}} \frac{\partial f}{\partial x_{j} \mid \mathbf{x}=\mathbf{x}^{0}}
$$

where $x_{j}^{0}$ is the $j$-th component of $\mathbf{x}^{0}$ and $y^{0}=$ $f\left(\mathbf{x}^{0}\right)$. 
For practical works, the partial derivative can mainly be computed by a finite difference scheme introducing a finite perturbation $\Delta x_{j}^{0}$ whose choice is delicate. The LSA is shown to be efficient for various problems [30, 31], but gives information only at the surrounding of the point $\mathbf{x}^{0}$. A bad choice of $\Delta x_{j}^{0}$ can cause a bad estimation of sensitivity in the whole domain if the response is non-linear, and cross-effects can not be observed. Even if $\Delta x_{j}^{0}$ is chosen to covers the entire domain, it can be easily shown that the ratio of the volume of the domain scanned by LSA methods (an hypersphere) to the volume of the real domain (assumed to be an hypercube) tends to zero when the number of dimensions $M$ of the domain grows. This is known as the OAT paradox.

At the opposite, Global Sensitivity Analysis (GSA) can manage automatically an exploration of the domain with the following properties:

- provided the input sample is of sufficient size, the entire domain is covered, there is no "OAT effect",

- parameters vary simultaneously: cross effects (i.e. interactions between inputs) can be detected,

- parameters vary throughout the whole domain: non linear effects are at least taken into account, but they can be precisely evaluated with appropriate tools.

\subsection{Principles of Global Sensitivity Analysis}

Global quantitative methods are based on performing many model evaluations in the whole range of the inputs, and using the results of these evaluations to determine how the variation of an input or a group of inputs contributes into the variation of output. To perform this global exploration, statistical methods based on Monte Carlo sampling are particularly well suited.

A global analysis on our model requires the generation of an input sample which can be stored into a $N \times M$ matrix $\mathbf{X}$, each line $\mathbf{x}_{i}$ of this matrix being a complete set of $M$ input parameters $x_{i j}$. As it can be seen later, different random processes can be used to do this sampling. After $N$ runs of the computer model using successively each line of $\mathbf{X}$, the $N$ outputs $y_{i}$ could be arranged in a $N$-dimensional vector $Y$.

From this point, each column $X_{j}$ of $\mathbf{X}$ and the output $Y$ can be seen as random variables for which

\begin{tabular}{cccccc}
\hline$T$ & $20{ }^{\circ} \mathrm{C}$ & $500{ }^{\circ} \mathrm{C}$ & $800{ }^{\circ} \mathrm{C}$ & $1100{ }^{\circ} \mathrm{C}$ & $1300{ }^{\circ} \mathrm{C}$ \\
\hline$E$ & $190 / 230$ & $148 / 185$ & $15 / 135$ & $5 / 30$ & $2 / 10$ \\
$\alpha$ & $9.4 / 17$ & $12 / 22.2$ & $11 / 23$ & $13 / 23.5$ & $14.4 / 24$ \\
$\nu$ & $0.22 / 0.32$ & $0.275 / 0.4$ & $0.319 / 0.464$ & $0.328 / 0.49$ & $0.34 / 0.498$ \\
\hline
\end{tabular}

Table 1: Material domain: ranges for three of the eight material properties. Each material property is defined at a discrete set of 5 temperatures which represent 15 input factors. Units: Young's modulus, E (GPa); thermal expansion coefficient, $\alpha\left(10^{-6} .{ }^{\circ} \mathrm{C}^{-1}\right)$; Poisson's ratio, $\nu(-)$

the model will be analyzed. Then a regression model for $Y$ using standard least squares analysis can be done. The standardized regression coefficients $S R C_{j}$ computation is then a first way to measure the sensitivity of $Y$ to the inputs $X_{j}$.

The three following sections are devoted to, firstly, the description of the material domain that we use in this study (section 3.4), secondly, the sampling procedures (section 3.5) and finally, a presentation of the considered sensitivity indices (section 3.6).

\subsection{Material domain}

In this study, the material domain was chosen to include the steel family. For our purpose, this domain is in fact the mathematical domain defined by the possible values of the model input parameters for all the steels. Of course this domain is not precisely known, and even if it was, it would be very difficult to represent. A convex envelope of this domain would be more convenient.

In fact, the hypercube envelope is the easiest way to include all materials. Of course this leads to consider in the study materials which have no real existence, but this can be tolerated for a domain including similar materials. This approach could be improper if used on a disparate family, including steels and aluminium alloys for example.

In table 1 the steel domain limits are shown for $3 \times 5=15$ of the $8 \times 5=40$ input parameters of the second study presented in section 4.2 .

\subsection{Sampling}

As it was noticed in section 3.3, the Global Sensitivity Analysis needs a sampling technique. The goal is to generate a set of experiments - each of them represented by a particular choice of input values. In the following subsection, three different techniques are presented.

The first two are classical ones, Simple Random Sampling (SRS) and Latin Hypercube Sampling (LHS). The third one is a particular adaptation of 
the LHS method to generate monotonic evolutions of material parameters in function of temperature. This new technique is called constrained Latin Hypercube Sampling (cLHS).

\subsubsection{Simple Random Sampling}

The most common sampling method is indisputably the Monte-Carlo or Simple Random Sampling (SRS), mainly because of its simplicity. It consists of randomly sampling $N$ independent input variables of $M$ material data. However, it is known to have slow rates of convergence (in $\mathcal{O}\left(N^{-1 / 2}\right)$ ): many code runs are required to obtain a satisfactory behaviour of statistical parameters of the response. An example of a SRS is presented on figure 1(a) in the case of two variables.

\subsubsection{Latin Hypercube Sampling}

Thirty years ago, Mc Kay, Conover and Beckmann 32] suggested an alternative method of generating the matrix of inputs $\mathbf{X}$ that they called Latin Hypercube sampling which is an extension of stratified sampling. LHS ensures that each of the input variables has all proportion of its range represented (in our case this method ensures that all areas of the steel sample space is sampled). Let the range of each $X_{j}, j=1 \ldots M$, be simultaneously partitioned into $N$ equally probable intervals, then a LHS of size $N$ is obtain from a random selection of $N$ values - one per stratum — for each $X_{j}$. Thus we obtain $M N$-tuples that form the $M$ columns of the $N \times M$ matrix of experiments $\mathbf{X}$ generated by LHS where the $i^{\text {th }}$ line contains the $M$ input variables $x_{i j}$ to perform the $i^{\text {th }}$ code execution. Once a point selected in an interval, no other point could be selected in this interval (represented by the red bands in the figure 1 (b) .

The partition into equally probable intervals is done to take into account non uniform densities of probability like a normal distribution for example (see figure 1 (b). In our case however, all parameters values in the domain interval are equiprobable. Hence, each interval for a given parameter is of same size.

Latin Hypercube Sampling ensures full coverage of the range of the input variables, which is not guaranteed with SRS. Sensitivity analysis results obtained with Latin Hypercube Sampling have been observed to be quite robust when small samples are used (only a few hundreds computer experiments for several tens of inputs). This explains the pop- ularity of this sampling method for use with longrunning models (see [33] for a review).

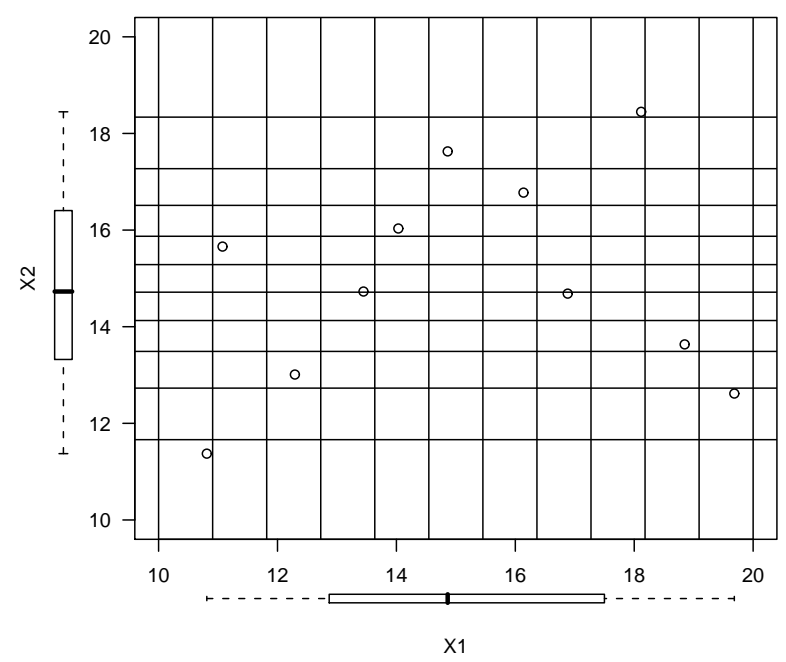

(a) Simple Random Sampling (SRS).

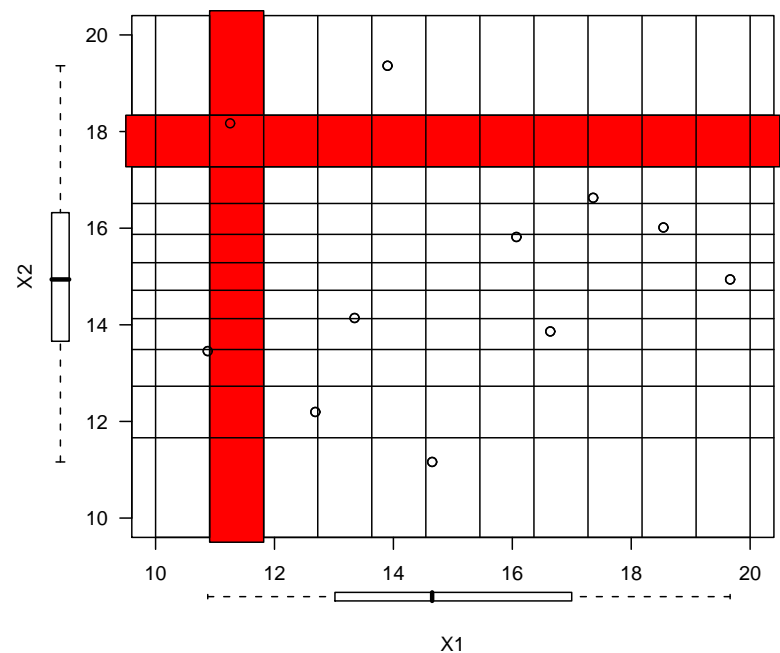

(b) Latin Hypercube Sampling (LHS).

Figure 1: Examples of two ways to generate a sample of size 11 from two variables $\mathbf{X}=\left[X_{1}, X_{2}\right]$ where $X_{1}$ has a uniform distribution and $X_{2}$ has a normal distribution.

\subsubsection{Conditional Latin Hypercube Sampling}

As it was seen before, parameter temperature evolutions are discretised for some chosen temperature values - typically 5 to 7 values. But the Finite Element code needs intermediate values during its execution. These intermediate values are computed by a piecewise linear interpolation between the discrete values. For physical reasons, it was decided that the discrete values - input parameters 
of the sensitivity analysis - corresponding to the temperature evolution of some material parameters must be constrained in order to give them monotonic variations. It is the case for Young's modulus for example, which is typically decreasing in function of the temperature for the steel family: a material with arbitrary Young's modulus variation could give non representative results for the studied phenomenon. On the opposite, other parameters like the thermal expansion coefficient for example, could have arbitrary variation versus temperature - according it belongs between the limits of the domain !

Hence, for the constrained parameters, a special sampling process is needed. An original method proposed in 34] consists in doing permutations on an initial LHS sample to respect the desired monotonic constraint. It is based on the fact that permuting two values of a variable in a LHS sample does not break the LHS structure of the sample. An appropriate algorithm scans the starting LHS sample to find the couples of values that violate the monotonic constraint. A criterion allows us to know if it exists a combination of permutations which is able to enforce the constraint. If it is not the case, a new LHS sample is created and the scan starts again. Fortunately, the process very often gives a positive result at the first try. Then the algorithm finds and executes the combinations of permutations which have to be done.

Figure 2 shows the work done on a couple of parameters on which an increasing constraint is enforced, and figure 3 shows the result on a 7-dimensional parameter with a decreasing constraint. Even if there is good reasons - physical and computational ones - to enforce these constraints, their precise action on the statistical behaviour of a model, in a more general case, is worth studying in future works.

\subsection{Estimate indices for the sensitivity analysis}

Various estimates of global sensitivity indices are available, among them Standardized Regression Coefficients (SRC), Pearson correlation coefficients, and Partial Correlation Coefficients (PCC). As these three estimates are used in this study, they are briefly presented below.

If the behaviour of $Y$ compared to each parameter is overall linear, it is possible to obtain quantitative measurements of their influence from the regression coefficients $b_{j}$ of the linear regression con-

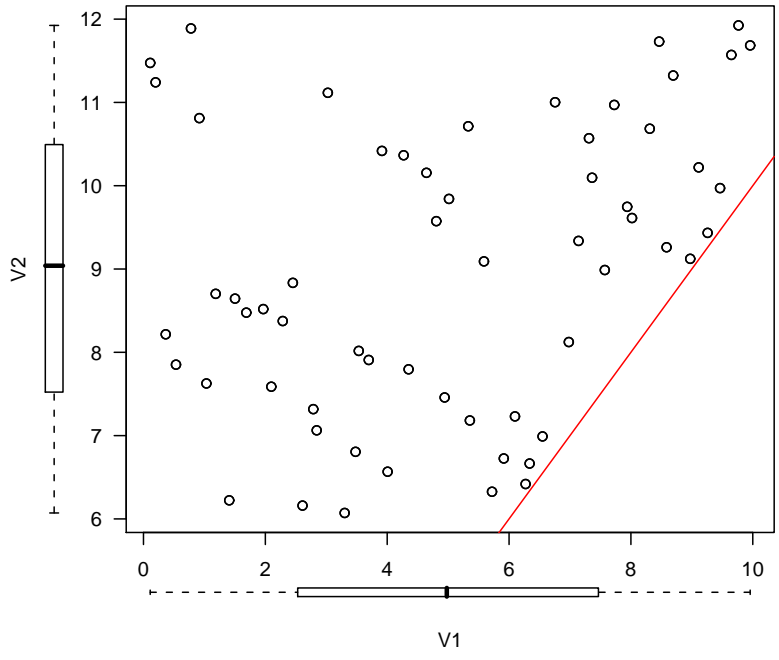

Figure 2: Example of constrained LHS sampling with an increasing constraint.

necting $Y$ to $X_{1}, X_{2}, \ldots, X_{M}$ :

$$
\hat{Y}=b_{0}+\sum_{j=1}^{M} b_{j} X_{j}
$$

The standardized regression coefficients are:

$$
S R C_{j}=b_{j} \cdot s_{X_{j}} / s_{Y}
$$

where $s_{X_{j}}$ and $s_{Y}$ are the respective standard deviations of $X_{j}$ and $Y$. These coefficients estimate the variation of the response for a given variation of a parameter $X_{j}$ and $S R C_{j}^{2}$ contains the part of the variance of the output $Y$ which is explained by $X_{j}$.

The Pearson product-moment correlation coefficient $\rho$ whose values belongs between -1 and 1 is helpful to estimate the strength of a linear relationship between two variables, taking values close than \pm 1 if the behaviour is linear. Unfortunately, this does not work for multi-variable analysis. In this case, one can build correlation matrices by computing the correlation coefficients of each pair of variables. This matrix can be useful to easily detect the more important parameters. Finally, $\rho^{2}$ is of the same order than $S R C^{2}$.

Here the Pearson correlation coefficients between each $X_{j}$ and $Y$ is written:

$$
\rho_{X_{j}, Y}=\frac{s_{X_{j} Y}}{s_{X_{j}} \cdot s_{Y}}
$$

$s_{X_{j} Y}$ being the covariance of $X_{j}$ and $Y$ :

$$
s_{X_{j} Y}=\frac{1}{N-1} \sum_{i=1}^{N}\left(x_{i j}-\overline{x_{j}}\right)\left(y_{i}-\bar{y}\right) .
$$


However, correlation between $Y$ and $X_{i}$ can be due to another variable (which is called "tierce correlation"). In that case, the SRC and the Pearson correlation coefficients are not pertinent. Partial correlation coefficients (PCC) estimate correlation between two variables when the others belongs constant (their effect is removed). The partial correlation coefficient between $Y$ and $X_{i}$ is the correlation coefficient between $Y-\widehat{Y}$ and $X_{i}-\widehat{X_{i}}$ :

$$
P C C_{j}=\rho_{Y-\widehat{Y}, X_{j}-\widehat{X_{j}}}
$$

where $\widehat{Y}$ is the linear regression of $Y$ when $X_{j}$ is not considered and $\widehat{X}_{j}$ is the linear regression of $X_{j}$ :

$$
\widehat{Y}=b_{0}+\sum_{\substack{h=1 \\ h \neq j}}^{M} c_{h} X_{h}, \quad \widehat{X_{j}}=d_{0}+\sum_{\substack{k=1 \\ k \neq j}}^{M} d_{k} X_{k}
$$

In our application case, using these PCC is relevant because the monotonic dependences between certain input parameters create some correlations between them. Therefore, SRC and Person coefficients are not protected against tierce correlations.

\section{Application to the numerical welding sim- ulation}

Two applicative studies are presented below. The first one is an academic $2 \mathrm{D}$ study whose aim was to develop and validate the entire process on a medium size problem. The second one is closer to a $3 \mathrm{D}$ classical industrial case, more complex and heavier in terms of computation time.

\subsection{First application: axi-symmetric heat deposit on a thick disc}

\subsubsection{Description}

The first application is a simple configuration, whose runtime allows several hundred code execution. Thus an axi-symmetrical test is led on a disc of diameter of $160 \mathrm{~mm}$ and height of $5 \mathrm{~mm}$ in order to investigate residual stresses and distortions generated by the heating process. This simulation which is quite similar that used by L. Depradeux [35] during his doctoral thesis give significant distortions without melting pool.

\subsubsection{Thermal computation}

As mentioned before (see section 21), the weak coupling allows to do the thermal computation before the mechanical one. The sensitivity analysis performed here does not concern the thermal part of the computation. Hence we obtain a single thermal loading which will be used in each mechanical simulation. Thermophysical properties (thermal diffusivity $\lambda$, density $\rho$, specific heat capacity $C_{p}$ ) must be described on a whole temperature range from room temperature up to $1200^{\circ} \mathrm{C}$. Their numerical values presented in the table 2 are taken from literature on the $316 \mathrm{~L}$. The heat input is modeled with a standard two-dimensional Gaussian function, and is applied at the center of the disk during a time of 120 s. The supplied power is $P=2500 \mathrm{~W}$.

\begin{tabular}{lcccccccccccc}
$\mathrm{T}\left({ }^{\circ} \mathrm{C}\right)$ & 20 & 100 & 200 & 300 & 400 & 500 & 600 & 700 & 800 & 900 & 1000 & 1200 \\
\hline$\lambda\left(\mathrm{W} / \mathrm{m}^{\circ} \mathrm{C}\right)$ & 14 & 15.2 & 16.6 & 17.9 & 19 & 20.6 & 21.8 & 23.1 & 24.3 & 26 & 27.3 & 29.9 \\
$\rho\left(\mathrm{Kg} / \mathrm{m}^{3}\right)$ & 8000 & 7970 & 7940 & 7890 & 7850 & 7800 & 7750 & 7700 & 7660 & 7610 & 7570 & 7450 \\
$C_{p}\left(\mathrm{~J} / \mathrm{Kg}^{\circ} \mathrm{C}\right)$ & 450 & 490 & 525 & 545 & 560 & 570 & 580 & 595 & 625 & 650 & 660 & 677 \\
\hline
\end{tabular}

Table 2: Thermophysical properties

\subsubsection{Mechanical computations}

In this study, an elastoplastic with kinematic linear hardening behaviour is considered (see section 2.2.2). Only 5 mechanical properties are considered: Young's modulus $E$, the thermal expansion coefficient $\alpha$, Poisson's ratio $\nu$, the yield strength $\sigma_{y}$ and the hardening modulus $H$. Their evolutions are discretised for 7 values of temperature. This lead to $7 \times 5=35$ parameters to be considered in the Sensitivity Analysis.

\subsubsection{Sampling}

To illustrate the sampling of the domain, we present in figures 3 and 4 examples for Young's modulus and for the thermal expansion coefficient. These curves present three randomly selected materials among the 800 created and the bounds of the domain. For the sensitivity analysis process, Young's modulus is represented by only 7 parameters, but one should keep in mind that for the mechanical computation, the curve represents truly the considered dependence of this modulus because the algorithm uses intermediate values according to a piecewise linear interpolation.

\subsubsection{Numerical aspects}

Thermo-mechanical simulations were performed using the finite element method and the 


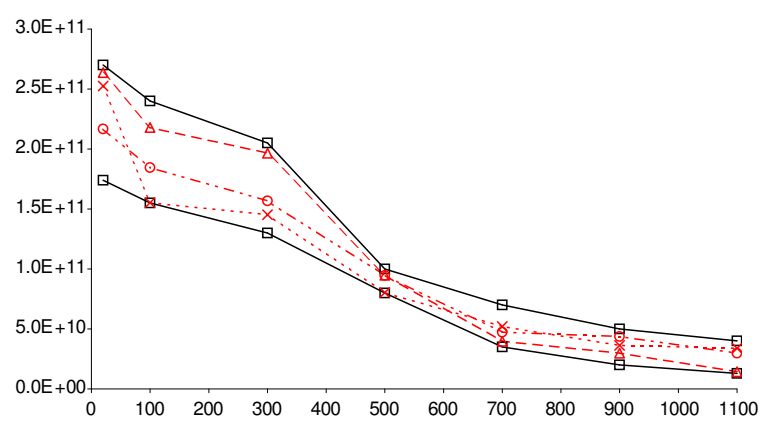

Figure 3: Example of constrained LHS sampling: Young's modulus of three randomly selected materials among the 800 created. The upper and the lower curves represent the bounds of the domain for these 7 parameters.

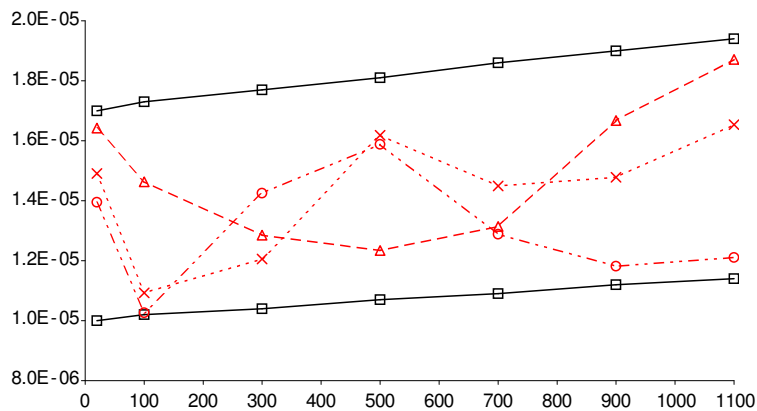

Figure 4: Example of (unconstrained) LHS sampling: thermal expansion coefficient of three randomly selected materials among the 800 created. The upper and the lower curves represent the bounds of the domain for these 7 parameters.
Cast3M 36 computer code. Simulations were performed with the 2D axi-symmetric FE mesh shown in figure 5. This mesh consists of 506 linear 4-node brick elements with 4 Gauss points. The central zone, where high thermal gradients are expected, is meshed with higher density. All the program settings (resolution algorithm, convergence criteria, time step...) are fixed for all the campaign.

As it can be seen later, 800 mechanical computations have been made. The complete computing time was about 25 hours on a 2004-model personal computer.

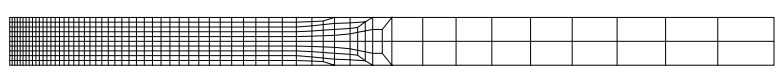

Figure 5: Axi-symmetric finite element mesh of the thick disk

\subsubsection{Choice of the sample size}

In order to verify the convergence of the Monte Carlo sample of the ouputs, an heuristic visualization tool is used: the mean value of each model output (associated with bootstrap estimates of their 95\%-confidence interval) are computed for a growing size set of samples. For example, the behaviour of the stored elastic energy is plotted in figure 6 . Similar resuts can be shown for other outputs. They show that beyond 600 samples, the mean value does not vary anymore. This shows that our sampling including 800 elements is representative for this study.

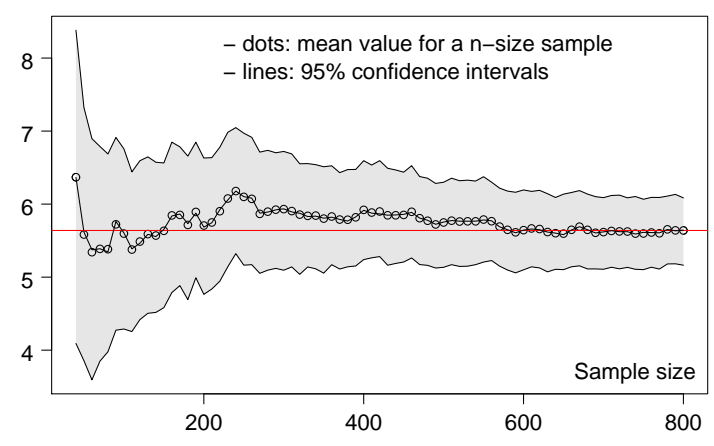

Figure 6: Thick disc study: Mean value and 95\% confidence interval of the ElastEner output.

\subsubsection{Results of the Sensitivity Analysis}

For this study, 4 outputs are chosen. The work of plastic deformation during the experiment, the 
stored elastic energy at the end of the experiment, the angle of the cone - which is approximately the shape of the deformed disc - at the end of the experiment and the maximum vertical displacement of the centre of the disc during the experiment. These 4 outputs are named respectively PlastWork, ElastEner, Angle and MaxUZ.

Table 3 presents linear sensitivity coefficients (SRC, Pearson and PCC) of the most important input parameters for the 4 chosen outputs. The squared coefficients are given in order to express the sensitivities in terms of explained variance. Only the ones with a $\mathrm{SRC}^{2}>0.02$ are shown in this table. The good value of $R^{2}$ tends to prove the linear behaviour of the studied output relatively to the inputs and gives to the sensitivity indices a good validity.

This sensitivity analysis shows that only three parameters among the 35 have a significant effect on the chosen outputs. They are: the yield strength at $20^{\circ} \mathrm{C}\left(\sigma_{y 20}\right)$, the thermal expansion coefficient at $20^{\circ} \mathrm{C}\left(\alpha_{20}\right)$, and Young's modulus at $20^{\circ} \mathrm{C}\left(E_{20}\right)$.

A difference should be noticed for the Max $\mathbf{U Z}$ output, the thermal expansion coefficient at $20^{\circ} \mathrm{C}$ has more influence than the yield strength at $20^{\circ} \mathrm{C}$. This is not illogical according to the fact that the MaxUZ output is quite particular because it is the only one among the four considered here which takes the maximum value of a quantity over the experiment duration. If the vertical displacement is plotted versus time, one must see the displacement goes at a much higher value than his final value. At this moment, temperature values are high all over the disk, giving a predominant weight to the thermal expansion coefficient. This is not the case for the three other outputs. It shows that the choice of the output is of great importance in such analysis.

The more surprising effect is that only 3 of the 35 parameters have a notable influence. To prove it a posteriori two series of 800 computations are made, the first one with random values for all the parameters (according to the procedure described above), the second with the same values for the three more influential parameters and mean values for the 32 other parameters. As it can be seen on the figure 7 despite the high variability of the considered output, the two series are almost equal (the $R^{2}$ is 0.9996 ). This result means that only 3 parameters have to be accuretly measured the 32 other parameters can be taken from classical litterature or arbitrary fixed at a meaning value. (a) PlastWork output - $\mathrm{R}^{2}=0.8327$

\begin{tabular}{lccc} 
& $\sigma_{y 20}$ & $\alpha_{20}$ & $E_{20}$ \\
\hline SRC $^{2}$ & 0.34 & 0.28 & 0.15 \\
Pearson $^{2}$ & 0.43 & 0.26 & 0.11 \\
PCC $^{2}$ & 0.43 & 0.62 & 0.31
\end{tabular}

(b) ElastEner output - $\mathrm{R}^{2}=0.9207$

\begin{tabular}{lccc} 
& $\sigma_{y 20}$ & $\alpha_{20}$ & $E_{20}$ \\
\hline SRC $^{2}$ & 0.42 & 0.32 & 0.14 \\
Pearson $^{2}$ & 0.48 & 0.3 & 0.12 \\
PCC $^{2}$ & 0.67 & 0.8 & 0.47
\end{tabular}

(c) Angle output - $\mathrm{R}^{2}=0.9445$

\begin{tabular}{lccc} 
& $\sigma_{y 20}$ & $\alpha_{20}$ & $E_{20}$ \\
\hline SRC $^{2}$ & 0.51 & 0.37 & 0.054 \\
Pearson $^{2}$ & 0.52 & 0.36 & 0.052 \\
PCC $^{2}$ & 0.78 & 0.87 & 0.33
\end{tabular}

(d) MaxUZ output - $\mathrm{R}^{2}=0.9361$

\begin{tabular}{lccc} 
& $\alpha_{20}$ & $\sigma_{y 20}$ & other \\
\hline SRC $^{2}$ & 0.8 & 0.13 & $<0.02$ \\
Pearson $^{2}$ & 0.79 & 0.12 & \\
PCC $^{2}$ & 0.92 & 0.44 &
\end{tabular}

Table 3: Sensitivity coefficients for the 4 outputs of the thick disc study.

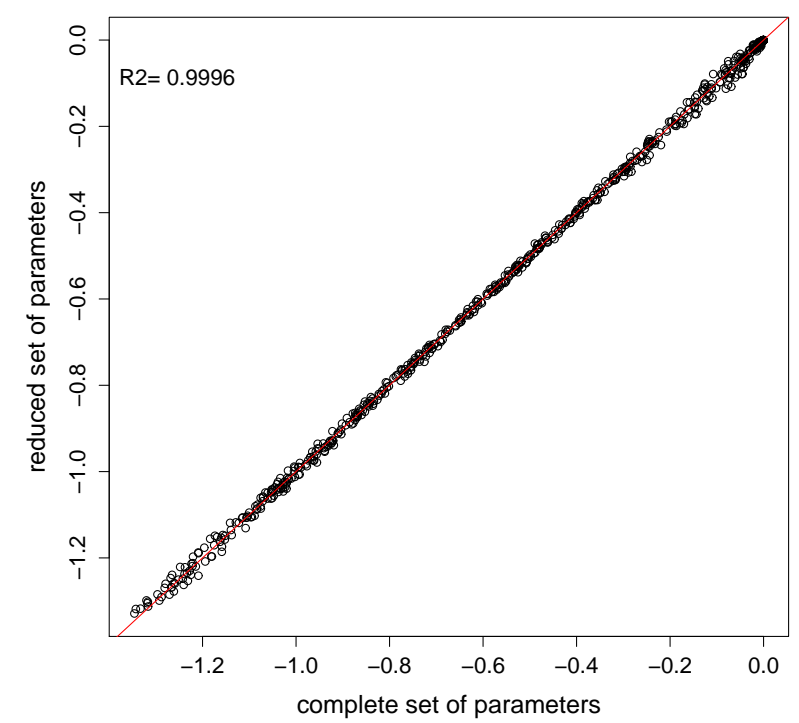

Figure 7: Thick disc study: Comparison of the output Angle for a $N=800$ LHS/cLHS sample between computations firstly made with a complete set of parameters and secondly with only the three more influential parameters (the 32 others are fixed to their mean value). 


\subsection{Second application: welding line on a thin plate}

\subsubsection{Description}

A square plate of side $250 \mathrm{~mm}$ and thickness $1.6 \mathrm{~mm}$ receives a line heat deposit simulating the use of a moving TIG source. The heat deposit is done over a limited path in the $x$-direction and the plate is clamped at its $x=x_{\max }$ side. This is done to limit computation time while keeping representative of a welding experiment.

According to the symmetry of the problem, only a half side of the plate is meshed. To reduce some more the computation time, a mixed mesh is used, see figure 8. This mesh is done with 3D elements in the heat deposit zone, and shell elements for the rest of the plate. So the problem is correctly modelised: the 4 layers of 3D elements in the heat deposit zone are able to manage strong thermal gradients and the viscoplasticity behaviour, unless the shell elements are sufficient to describe the thermomechanical bending of the rest of the plate because the viscoplasticity is absent and the thermal variation in the $z$-direction is moderate.

This mesh is made up of 3934 elements, 2760 8nodes hexahedrons and 1174 3-nodes DKT shell elements. Special conditions are prescribed at the interface between solid and shell elements to verify forces equilibrium and heat flux balance.

The heat deposit is modelised by a volumetric heat source model proposed by Goldak [37]. The seven parameters used by this model are classically adjusted by inverse methods from experiment results.

The mechanical model is the viscoplastic model described in section 2.2.2 with 8 parameters. In order to limit the computation time, each of the 8 parameters temperature evolutions are discretised only for the 5 temperature values: $20,500,800$, 1100 and $1300{ }^{\circ} \mathrm{C}$. This leads to 40 input parameters for the sensitivity analysis, which is comparable to the precedent study.

However, this 3D finite element model is bigger than the above 2D disk model: time computation for one set of parameters is now about 6 hours. As a 500 materials sampling had been considered for this study, the total time computation is estimated to 125 days. Fortunately, this type of study, which is by nature highly parallelizable, was made using several computers.

Other numerical aspects are identical to those described above.

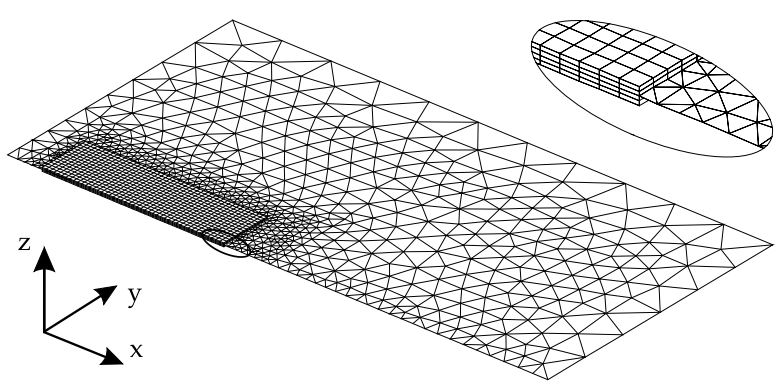

Figure 8: Thin plate study: Mesh of the plate.

\subsubsection{Sensitivity analysis}

In this study, it was decided to consider only displacements outputs. Three points were chosen on the plate and their vertical displacement $u_{z}$ were followed up during each experiment. As their evolutions were similar, only one of them will be considered here. This point $P$ is the $\left(x_{\min }, y_{\max }\right)$ corner, at the opposite of the clamped side. Only final values are taken for the sensitivity analysis, but the evolution is particularly interesting as can be seen in figure 9

This figure shows, for all the experiments, the time evolution of the $u_{z}$ displacement of the considered point. It can be seen that some experiments give "opposite" results. From a mechanical point of view, this phenomenon seems to resemble a buckling instability. The structure hesitates to bend in a direction or in the opposite direction. For a very small change in some properties, the final state can be very different. As the purpose of this study was to implement the sensitivity analysis, this particular aspect is no more developed here, even if it seems particularly interesting. So, we choose to ignore these "opposite" results because they can produce strong artificial sensitivity related to this - presumed - elastic instability and would lead to bad results. That points out the great importance of output choice.

The result of the sensitivity analysis is presented on figure 11] This figure shows that the result is different from the precedent study except from the fact that three of the forty parameters share 90 percent of the variability of this output. In this case however, the most influential parameters are high-temperature values of the plastic strain coefficient $A_{1300}$ Young's modulus $E_{1300}$ and the thermal expansion coefficient $\alpha_{1300}$. Numerical values are given in table 4.

The fact that the result is different from the 


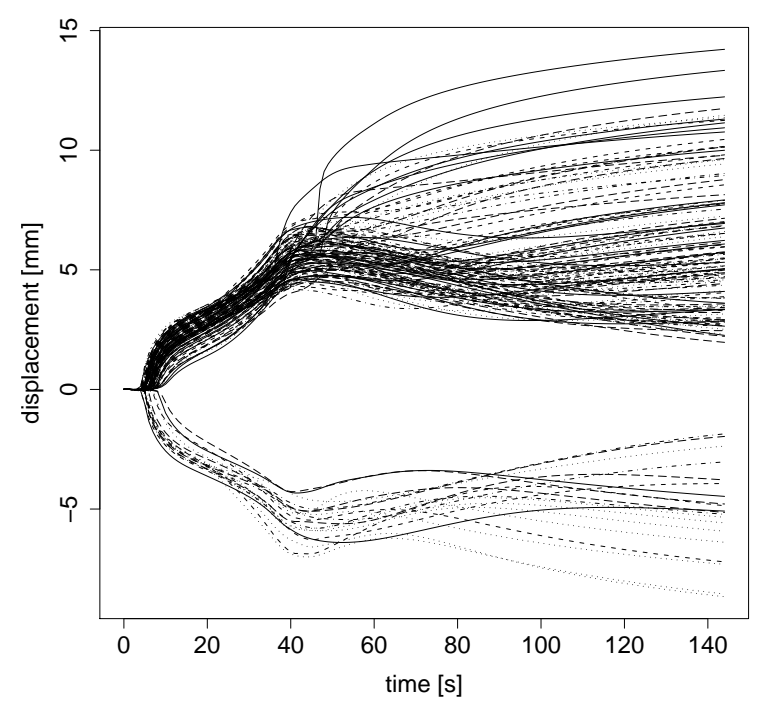

Figure 9: Thin plate study: Displacement curves for each material simulated (vertical displacement on the point $P$ ).

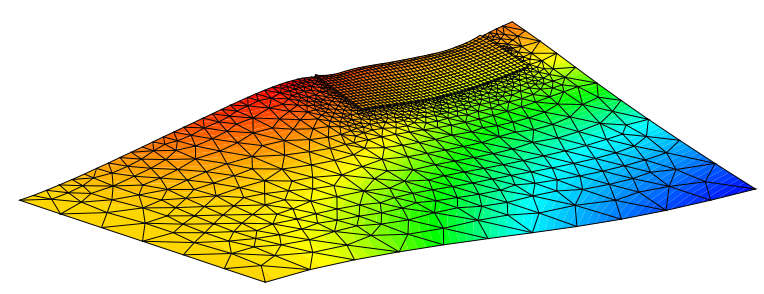

Figure 10: An example of deformed plate precedent study is due to the nature of the problem. This will be discussed in the section 4.3 .

\begin{tabular}{|c|c|c|c|}
\hline \multicolumn{2}{|c|}{ Final displacement $z$} & \multicolumn{2}{|c|}{$-\mathrm{R}^{2}=0.9004$} \\
\hline $\mathrm{SRC}^{2}$ & 0.41 & 0.25 & 0.068 \\
\hline Pearson $^{2}$ & 0.34 & 0.18 & 0.24 \\
\hline $\mathrm{PCC}^{2}$ & 0.73 & 0.63 & 0.062 \\
\hline
\end{tabular}

Table 4: Thin plate study: Sensitivity coefficients for the final $z$-displacement of the point $P$.

\subsection{Discussion}

The two studies gave different results. The first one has shown that low temperature parameters $\sigma_{y 20}, \alpha_{20}$, and $E_{20}$ were the most important. The second study leads to a different ranking, the most influential are the high temperature parameters: $A_{1300}, E_{1300}$ and $\alpha_{1300}$.

These two problems are different for many reasons. Firstly, the model is elasto-plastic in the disk study and elasto-viscoplastic in the plate study. As the sets of parameters are not the same in the two studies, even the common parameters may have different relative importance in each model. Secondly, the nature of the two studies strongly differs. The disk is thick and solicited at his centre. It is a very rigid structure. When the disk has cooled down, only a small plastic zone at the centre struggles against the rest of the disk which remains elastic. In the plate case, the plate is thin and easy to bend, and the solicitation is more extended than a point, it is a line. This is a less rigid case. Further, because the plate is thin, all the heated zone has reached high temperatures, which may explain the high temperature parameters ranking.

These two studies show that the validity of the sensitivity analysis result is attached to the considered system or model. General conclusions must be very carefully tested before their confirmation. They also show the power of the sensitivity analysis tool for numerical simulations like welding, allowing strong reduction of models - provided the respect of the precaution mentioned above.

\section{Conclusion}

The present paper has suggested an efficient procedure to perform a sensitivity analysis in welding simulation. The study was limited to the sensitivity to mechanical parameters. The unsteady thermal 


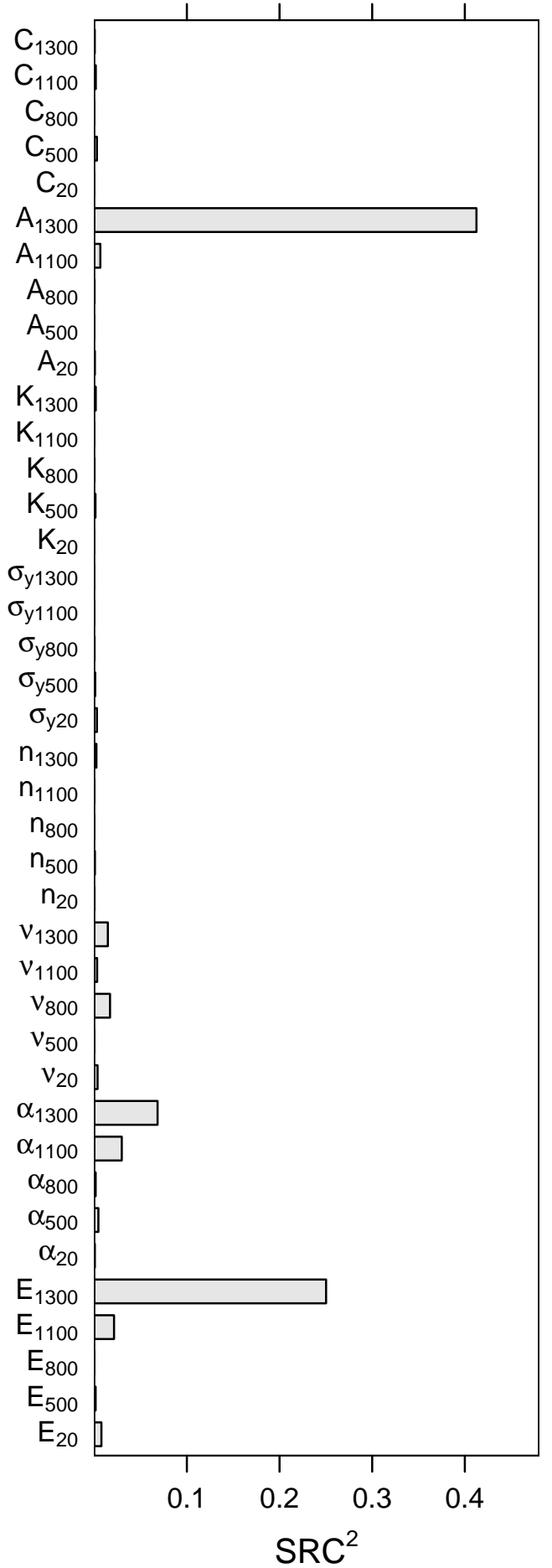

Figure 11: Thin plate study: Final $z$-displacement of the point $P$. Sensitivity indexes $\mathrm{SRC}^{2}$ for the 40 inputs $(8 \mathrm{me}-$ chanical properties discretized for 5 temperature values) response was computed only once, and the temperature field evolution used as thermal load in mechanical computations.

The sensitivity analysis was chosen to be of global type, in order to covers the entire steel domain without the drawbacks of local methods. Inputs are the parameters of the mechanical models, each of them discretised into values for different temperatures. This leads to a set of 35 (40) inputs, which is quite a lot dealing with sensitivity analysis. Outputs are classical mechanical observables like displacements, stresses or energy. A LHS technique generates a sample of input parameters set, each of them representing a virtual steel.

Physical considerations have lead to modify the classical LHS technique to enforce monotonic temperature variation for some of the mechanical parameters. This constrained LHS technique proposed in [34] was used to sample these particular mechanical parameters, as a classical LHS technique was used for the others.

Numerical welding simulations are performed for all the virtual materials of the sample, and the sensitivity analysis is done. Sensitivity indices like Standardized Regression Coefficient (SRC), Pearson correlation coefficients and Partial Correlation Coefficients (PCC) are computed for all input parameters.

Two practical studies were achieved. These two studies differ in the welding configuration and considered mechanical models (one is elastoplastic with 35 parameters and the other is elastoviscoplastic with 40 parameters). The results of the two sensitivity analysis are different but, a surprising fact is that, in both cases, only 3 of the 35 (40) input parameters explains $90 \%$ of the outputs variability.

To verify a posteriori this result, a sample of a 800 materials is generated. A duplicata of this sample is made, keeping the random values for the 3 most important parameters and giving mean values to all the other parameters. Two series of computations were performed, one for the native sample and one for the degenerated one. Then outputs were compared for both corresponding materials. Which is remarkable is, despite the high variability of the considered outputs, both couples of corresponding materials had given very near results.

Sensitivity analysis has provided answers to what we consider one of the probable frequently asked questions regarding welding simulation: for a given welding problem, which properties must be measured with a good accuracy and which ones can be 
simply extrapolated or taken from a similar material? Indeed, a Global Sensitivity Analysis performed onto a representative numerical model is able to find the most influent input parameters. Considering the preliminary results of this work it is permitted to think that they are very few compared to all the required inputs. That may avoid doing numerous difficult and expensive experiments to determine precisely parameters whose mean value is proved to be sufficient.

Finally, a new simulation methodology is proposed, including four sequential steps: Firstly the problem needs to be characterized (nature, material, models, inputs and outputs, domain, etc.) and a representative numerical model is built. Secondly, a Global Sensitivity Analysis is done, which leads to the ranking of input sensitivities. Then the most influential parameters are carefully measured -if possible - on the considered material, other material properties are fixed to probable values (representative of the material family for example). Numerical simulations can now be performed. Three advantages could be expected: a substantial reduction of the amount of experiments and the induced financial and time economies, more accurate results because precise data are considered for the most influential parameters, the physical meaning of the sensitivity analysis results can lead to a better understanding of phenomena.

\section{References}

[1] A. Loredo, B. Martin, H. Andrzejewski, and D. Grevey. Numerical support for laser welding of zinc-coated sheets process development. Applied Surface Science, 195(1-4):297-303, July 2002.

[2] B. Martin, A. Loredo, D. Grevey, and A.B. Vannes. Numerical investigation of laser beam shaping for heat transfer control in laser processing. Lasers in Engineering, 12(4):247-269, 2002.

[3] J. K. Hepworth. Finite element calculation of residual stresses in welds. Num. Meth. Non-Lin. Prob., pages 51-60, 1980.

[4] P. Tekriwal and J. Mazumder. Transient and Residual Thermal Strain-Stress Analysis of GMAW. Journal of Engineering Materials and Technology, 113:336-343, 1991.

[5] L.E. Lindgren. Finite element modeling and simulation of welding part 3: efficiency and integration. Journal of thermal stresses, 24:305-334, 2001.

[6] J. Canas, R. Picon, F. Pariis, A. Blazquez, and J. C. Marin. A simplified numerical analysis of residual stresses in aluminum welded plates. Computers \& 8 Structures, 58(1):59-69, January 1996.

[7] G. Chen, X. Xu, C. C. Poon, and A. C. Tam. Experimental and 2D numerical studies on microscale bending of stainless steel with pulsed laser. Journal of Applied Mechanics, 66:772-779, 1999.

[8] Q. Y. Shi, A. L. Lu, H. Y. Zhao, P. Wang, A. P. Wu, Z. P. Cai, and Y. P. Yang. Effects of material properties at high temperature on efficiency and precision of numerical simulation for welding process, volume 2 of ISBN: 0965700135, chapter Advances in Computational Engineering \& Sciences, pages 655-660. 2000.

[9] K. Abdel-Tawab and A. K. Noor. Uncertainty analysis of welding residual stress fields. Computer Methods in Applied Mechanics and Engineering, 179(3-4):327-344, September 1999.

[10] X. K. Zhu and Y. J. Chao. Effects of temperaturedependent material properties on welding simulation. Computers \& Structures, 80(11):967-976, May 2002.

[11] J. Song, J. Y. Shanghvi, and P. Michaleris. Sensitivity analysis and optimization of thermo-elasto-plastic processes with applications to welding side heater design. Computer Methods in Applied Mechanics and Engineering, 193(42-44):4541-4566, October 2004.

[12] M. Petelet and O. Asserin. Influence des paramètres d'un modèle élastoplastique sur les distorsions calculées par simulation numérique d'une opération de soudage. In Congrès Francais de la Mécanique, 2005.

[13] C. Schwenk, M. Rethmeier, K. Dilger, and V. Michailov. Sensitivity analysis of welding simulation depending on material properties value variation (submitted). In Mathematical Modelling of Weld Phenomena, volume 8, Graz, 2007.

[14] D. A. Tortorelli and P. Michaleris. Design sensitivity analysis : Overview and review. Inverse Problems in Engineering, 1:71-103, 1994.

[15] M. D. Morris. Factorial sampling plans for preliminary computational experiments. Technometrics, 33(2):161174, May 1991.

[16] J. C. Helton. Uncertainty and sensitivity analysis techniques for use in performance assessment for radioactive waste disposal. Reliability Engineering and System Safety, 42:327-367, 1993.

[17] A. Saltelli, T. H. Andres, and T. Homma. Sensitivity analysis of model output: An investigation of new techniques. Computational Statistics 83 Data Analysis, 15:211-238, 1993.

[18] J.P.C. Kleijnen. Sensitivity analysis and related analyses: a review of some statistical techniques. Journal of Statistical Computation and Simulation, 57:111-142, 1997.

[19] B. Iooss, F. Van Dorpe, and N. Devictor. Response surfaces and sensitivity analyses for an environmental model of dose calculations. Reliability Engineering 83 System Safety, 91:1241-1251, 2006.

[20] A. Saltelli, K. Chan, and E. M. Scott. Sensitivity Analysis. Wiley, 2000.

[21] A. Saltelli, S. Tarantola, F. Campolongo, and M. Ratto. Sensitivity Analysis in Practice: A Guide to Assessing Scientific Models. WILEY, 2004. 232 pages.

[22] K. T. Fang, R. Li, and A. Sudjianto. Design and Modeling for Computer Experiments, volume 6. Chapman \& Hall/CRC Computer Science \& Data Analysis, 2005.

[23] E. De Rocquigny, N. Devictor, and S. Tarantola, editors. Uncertainty in industrial practice. Wiley, 2008.

[24] B. Iooss. Manuel utilisateur du logiciel SSURFER V1.2 : programmes en $\mathrm{R}$ d'analyses d'incertitudes, de sensibilités, et de construction de surfaces de réponse. Technical report, NOTE TECHNIQUE CEA, 
DEN/CAD/DER/SESI/LCFR/NT DO 6 08/03/06, 2006.

[25] R Development Core Team. R: A Language and Environment for Statistical Computing. R Foundation for Statistical Computing, Vienna, Austria, 2006. ISBN 3-900051-07-0.

[26] P. Pilvin. Identification des paramètres de modèles de comportement. In MECAMAT, pages 155-164, Besançon, 1988.

[27] J.L. Chaboche. A review of some plasticity and viscoplasticity constitutive theories. International Journal of Plasticity, 24(10):1642 - 1693, 2008. Special Issue in Honor of Jean-Louis Chaboche.

[28] L. E. Lindgren. Modelling for residual stresses and deformations due to welding - Knowing what isn't necessary to know. In H. Cerjak, editor, Mathematical Modelling of Weld Phenomena, volume 6, 2002. presented in Graz, Austria, Oct 2001.

[29] A. Saltelli, M. Ratto, T. Andres, F. Campolongo, J. Cariboni, D. Gatelli, M. Saisana, and S. Tarantola. Global Sensitivity Analysis: The Primer. WILEY, 2008. 304 pages.

[30] D. G. Cacuci. Sensitivity theory for non-linear systems. Journal of Mathematical Physics, 22, 1981.

[31] T. Turanyi. Sensitivity analysis of complex kinetic systems. tools and applications. Journal of Mathematical Chemistry, 5(3):203-248, September 1990.

[32] M. D. Mc Kay, W. J. Conover, and R. J. Beckman. A comparison of three methods for selecting values in the analysis of output from a computer code. Technometrics, 21(2):239-245, 1979.

[33] J. C. Helton and F. J. Davis. Latin hypercube sampling and the propagation of uncertainty in analyses of complex systems. Reliability Engineering \& System Safety, 81(1):23-69, July 2003.

[34] M. Petelet. Analyse de sensibilité globale de modèles themomécaniques de simulation numérique du soudage. PhD thesis, University of Burgundy, 2007.

[35] L. Depradeux. Simulation numérique du soudage Acier 316L. Validation sur cas tests de complexité croissante. PhD thesis, INSA Lyon, 2003.

[36] Cast3M. CEA Finite Element software, 2007.

37] J. Goldak, A. Chakravarti, and M. Bibby. A new finite element model for welding heat flow in welds. Metallurgical Transaction B, 15B:299-305, June 1984. 